\title{
APLICAÇÃO DO SISTEMA DE INDICADORES DE QUALIDADE AMBIENTAL PARA METRÓPOLES COSTEIRAS (SIMEC): A REGIÃO DE JOINVILLE- SC COMO ESTUDO DE CASO
}

Resumo: O presente artigo consiste na aplicação do Sistema de Indicadores de Qualidade Ambiental Urbana para Metrópoles Costeiras (SIMeC) na Região de Joinville-SC. O SIMeC foi elaborado a partir do levantamento de 1.133 indicadores já existentes em diferentes regiões abrangendo Europa, Ásia, América e Oceania. Após uma primeira análise que resultou em 32 indicadores, eles foram avaliados por 45 especialistas de todos os estados costeiros brasileiros que indicaram novos indicadores para compor o sistema. Como resultado o sistema consiste em 69 indicadores organizados por dimensões que possibilitam uma análise sistêmica. Com a aplicação na Região de Joinville, é possível observar as ações de planejamento urbano realizadas, assim como a necessidade de investimentos para a promoção das melhorias de serviços e equipamentos urbanos da região.

Palavras-chave: Indicadores. Qualidade Ambiental Urbana. Prosperidade Urbana. DPSIR. Região de Joinville-SC.

\section{THE APPLICATION OF THE URBAN ENVIRONMENTAL QUALITY INDICATOR SYSTEM FOR COASTAL METROPOLIS: IN THE JOINVILLE-SC REGION}

Abstract: This article describes the application of the Urban Environmental Quality Indicator System for Coastal Cities (SIMeC) in the Joinville-SC Region. SIMeC was drawn from the survey of 1.133 indicators that already existed in different regions abroad Europe, Asia, Americas and Oceania. Which after a first analysis which resulted in 32 indicators, were evaluated by 45 experts from all Brazilian coastal states, who indicated new indicators to make up the system. As a result, the system consists of 69 indicators organized by dimensions which allow a systemic analysis. As a consequence of the application in the Joinville region, it is possible to observe the urban planning actions taken, as well as the investment needs in order to promote service improvements and urban infrastructure in the region.

Keywords: Indicators. Urban environmental quality. Urban Prosperity. Joinville Region.

\section{LA APLICACIÓN DE UN SISTEMA DE INDICADORES DE CUALIDAD AMBIENTAL URBANA PARA METRÓPOLIS COSTERAS (SIMEC): LA REGIÓN DE JOINVILLE - SC CÓMO ESTUDIO DE CASO}

Resumen: El presente artículo está basado en la aplicación de un Sistema de Indicadores de Cualidad Ambiental Urbana para Metrópolis Costeras (SIMeC) en la región de Joinville - SC. EI SIMeC ha sido elaborado a partir de levantamiento de indicadores existentes en distintas regiones de Europa, Asia, América y Oceanía.

\footnotetext{
1 Universidade Estadual de Ponta Grossa (UEPG), Departamento de Geociências, Ponta Grossa, Brasil, niltonadao@hotmail.com, https://orcid.org/0000-0002-6387-2004

2 Universidade do Vale do Itajaí (UNIVALI), Programa de Pós-Graduação em Ciência e Tecnologia Ambiental, EMCT - Laboratório de Conservação e Gestão Costeira, Itajaí, Brasil, mpolette@univali.br, https://orcid.org/0000-0003-0437-4205
} 
Después de un primer análisis fueron evaluados por 45 especialistas de todos los estados costeros brasileños que indicaran nuevos indicadores para componer el sistema. Como resultado el sistema consiste en 69 indicadores ordenados por dimensiones que posibilitan un análisis sistémico. Con la aplicación en la región de Joinville es posible observar que las acciones de planeamiento urbano realizadas, bien como las necesidades de inversiones para promover las mejorías de servicios y equipamientos urbanos de la región.

Palabras-clave: Indicadores. Cualidad Ambiental Urbana. Prosperidad Urbana. DPSIR. Región de Joinville - SC.

\section{Introdução}

Nas últimas décadas é possível encontrar muitas ações para gestão e entendimento das dinâmicas costeiras. No entanto, a melhor definição da extensão geográfica para a gestão sustentável e engajamentos das partes interessadas continua um desafio (SREEJA; MADHUSOODHANAN; ELDHO, 2016). Diante disso, este artigo apresenta um sistema de indicadores que delimita a análise dos diferentes fenômenos que envolvem a realidade costeira tendo como território os aglomerados urbanos e as regiões metropolitanas aplicado na Região de JoinvilleSC.

No Brasil, já são previstos instrumentos de apoio à gestão como, por exemplo, a publicação periódica de Relatórios de Qualidade Ambiental da Zona Costeira (RQA-ZC) para monitorar e avaliar as ações de gestão costeira integrada (DECRETO 5300/2004). No entanto, os RQA-ZC, de competência de cada Poder Público Estadual, ainda não se consolidaram como documentos de referência para diagnosticar as zonas costeiras. Ao mesmo tempo, apesar da existência relevante de estudos acadêmicos, produções associadas aos instrumentos oficiais de gestão costeira ainda são incipientes. Considerando esta lacuna, o presente artigo apresenta uma ferramenta para monitoramento e análise das regiões metropolitanas costeiras para avaliar a qualidade ambiental urbana nas diferentes dimensões da sustentabilidade.

Para tanto, parte-se do pressuposto que entender os padrões de uso e ocupação populacionais perpassa pelo entendimento da qualidade dos serviços ofertados e do padrão de gestão que indicam a qualidade ambiental urbana (KAMP; et. al., 2003; FENERI, VAGIONA, KARANIKOLAS, 2003; PACIONE, 2003; LAGO, 2015) que assume um caráter multimensional, que Kamp et. al. (2003) associaram a uma cebola por conta das suas múltiplas camadas. Nesse caso, os indicadores se 
apresentam como instrumentos relevantes para o entendimento de diferentes variáveis e dimensões que configuram esses espaços.

Quando consistentes, os indicadores informam os status e estabelecem referenciais e tendências para tomadas de decisão (COLL, et. al. 2016; BEROYAEITNER, 2016). De forma geral os mesmos são cada vez mais aplicados em diferentes abordagens (BEROYA-EITNER, 2016; COLL, et. al. 2016; WANG, et. al. 2014; LOZANO, GRANADOS, GUZMÁN, 2014) e, neste estudo, os indicadores associam a qualidade ambiental urbana com a realidade de áreas urbanas costeiras.

Diante do exposto, neste artigo é aplicado o Sistema de Indicadores para Metrópoles Costeiras (SIMeC), cujo detalhamento do método é encontrado em Adão e Polette (2016). De maneira resumida, o método de construção dos indicadores consistiu em, inicialmente, levantar e selecionar indicadores já existentes considerando a relevância para temática. Ao total foram analisados 1.133 indicadores de 22 sistemas de análise urbana ou de análise das zonas costeiras aplicados em diferentes países da América, Ásia, Oceania e Europa. Realizou-se uma análise inicial compondo uma versão preliminar dos indicadores considerados mais pertinentes que, posteriormente, foram validados por uma equipe multidisciplinar de 45 pesquisadores atuantes nos 17 estados costeiros do Brasil. Os especialistas também indicaram novos indicadores que foram integrados ao sistema diante do atendimento aos critérios: relevância; factibilidade operacional; relação sistêmica e a não sobreposição com os indicadores já existentes (ADÃO; POLETTE, 2016).

Na sua organização, os indicadores são apresentados em cinco estruturas de DPSIR de acordo com as dimensões da Prosperidade Urbana do Índice de Prosperidade das Cidades (IPC). O IPC, apresentado no Relatório Stateof World's Cities 2012/13: Prosperity of Cities (UN-HABITAT, 2012), objetiva promover a prosperidade além da já tradicional prosperidade econômica incluindo como dimensões, além da Produtividade, a Infraestrutura, Qualidade de Vida, Inclusão e Equidade Social e a Sustentabilidade Ambiental. Nesse contexto, a prosperidade de uma cidade está associada à forma como ela é abrangente e acessível a todos. Assim, a cidade mais próspera é caraterizada pelo crescimento econômico inclusivo, promoção da diversidade social, preocupação com o ambiente natural e a redução das vulnerabilidades sociais e ecológicas (UN-HABITAT, 2012).

Ao se apropriar das dimensões da Prosperidade Urbana, o presente artigo busca compreender a qualidade ambiental urbana, não na forma de índice, mas sob 
a ótica de indicadores para medir e analisar o estado das cidades que compõe uma região costeira. Para tanto parte-se da premissa que, no contexto das regiões e metrópoles costeiras, o entendimento da qualidade ambiental urbana, perpassa por espaços vulneráveis diante dos usos e ocupações humanas e fluxos de toda ordem que impactam nos ambientes naturais (NICOLODI; ZAMBONI, 2009; POLETTE, LINS-DE-BARROS, 2012; PADILLA, 2013). Assim, para mensurar as vulnerabilidades e a satisfação pessoal nos ambientes costeiros também se deve considerar o sistema urbano em todas as dimensões da qualidade ambiental.

Para validar o seu potencial, o presente artigo tem como objetivo aplicar o Sistema de Indicadores de Qualidade Ambiental Urbana para Metrópoles Costeiras - SIMeC - na Região de Joinville-SC. Um aglomerado urbano que, apesar de oficialmente não ser uma região metropolitana, apresenta um processo de urbanização caracterizada pela industrialização ainda eminente em uma região costeira com características socioambientais que ainda carecem de entendimento e de uma análise que contextualize as relações e necessidades dos municípios que a compõem.

Como característica, os 69 indicadores não são apresentados como índice valorizando as especificidades de cada indicador e permitindo também uma análise integrada. Para ampliar a análise, os 69 indicadores divididos nas dimensões da Prosperidade Urbana, estão classificados de acordo com a relação causal entre forças-motrizes, pressões, estados, impactos e respostas (Drivers-PressuresState-Impacts-Responses) conhecido como DPSIR. Segundo Svarstad et. al. (2008), uma estrutura amplamente aceita por pesquisadores e formuladores de políticas ao capturar e informar as relações entre a sociedade e o meio ambiente (ADÃO; POLETTE, 2016; ADÃO; POLETTE, 2018).

\section{Aplicação do SIMeC Região de Joinville-SC}

A Região de Joinville- SC, localizada no Norte/Nordeste catarinense apresenta uma faixa terrestre que integra os municípios costeiros de Joinville, Araquari, São Francisco do Sul, Itapoá, Garuva, Barra Velha e Balneário Barra do Sul compondo, segundo o Plano Estadual de Gerenciamento Costeiro, o Setor 1 localizado no litoral norte de Santa Catarina. Por uma questão de recorte territorial também foi considerado neste estudo, o município não costeiro de São João do Itaperiú localizado entre os munícipios de Barra Velha e Araquari. 
De acordo com o diagnóstico do GERCO-SC (2010), o Setor 1 do litoral norte catarinense apresenta diferentes atividades industriais (ainda em expansão) e de ocupação que, na integração entre o social no espaço natural, exigem um modelo próprio de compreensão e atuação por apresentar cenários que são produtos de um desenvolvimento, pouco ou nada planejado. Para analisar a realidade da região os indicadores serão apresentados para cada município por meio das dimensões da Prosperidade Urbana, sendo constituída uma estrutura DPSIR para cada dimensão.

\section{Qualidade de Vida}

Nas cidades mais desiguais, a qualidade de vida está fortemente associada com o fornecimento de bens públicos sob a forma de serviços básicos para atender as populações que se concentram nas áreas urbanas (UN-HABITAT, 2012). O quadro 1 apresenta o resultado desta dimensão.

Quadro 1- Região de Joinville e a dimensão Qualidade de vida

\begin{tabular}{|c|c|c|c|c|c|c|c|c|c|}
\hline DPSIR & \multicolumn{8}{|c|}{ Aplicação dos indicadores } & Fonte \\
\hline \multirow{3}{*}{$D$} & \multicolumn{8}{|c|}{ Taxa estimada de crescimento da população } & \multirow{3}{*}{$\begin{array}{l}\text { IBGE } \\
\text { (2015) }\end{array}$} \\
\hline & AR & BS & BV & GA & IT & $\mathrm{JO}$ & SF & SJ & \\
\hline & 4,6 & 2,6 & 2,8 & 2,1 & 3,5 & 1,3 & 2,2 & 0,7 & \\
\hline \multirow{3}{*}{$P$} & \multicolumn{8}{|c|}{ Densidade demográfica } & \multirow{3}{*}{$\begin{array}{l}\text { IBGE } \\
(2010)\end{array}$} \\
\hline & AR & BS & BV & GA & IT & $\mathrm{JO}$ & SF & SJ & \\
\hline & 64 & 75 , & 159 & 29 & 59 , & 457 & 85 , & 22,69 & \\
\hline \multirow{3}{*}{$S$} & \multicolumn{8}{|c|}{ Taxa de motorização (automóvel para cada 100} & \multirow{3}{*}{$\begin{array}{l}\text { Feco- } \\
\text { mércio } \\
(2012)\end{array}$} \\
\hline & AR & BS & BV & GA & IT & $\mathrm{JO}$ & SF & SJ & \\
\hline & 15 & 15, & 20 & 20 & 12 & 34 & 20 & 23,8 & \\
\hline S & & & tensã & da re & le de & siclov & is em & & $\begin{array}{c}\text { Prefeituras } \\
(2015)\end{array}$ \\
\hline \multirow{3}{*}{$S$} & \multicolumn{8}{|c|}{ Número de médicos por mil habitantes } & \multirow{3}{*}{$\begin{array}{l}\text { Datasus } \\
(2013)\end{array}$} \\
\hline & AR & BS & BV & GA & IT & $\mathrm{JO}$ & SF & SJ & \\
\hline & 1,1 & 0,5 & 0,6 & 0,9 & 0,6 & 2,1 & 0,8 & 0,56 & \\
\hline \multirow{3}{*}{ S } & \multicolumn{8}{|c|}{ Número de leitos hospitalares por mil habitantes } & \multirow{3}{*}{$\begin{array}{l}\text { Datasus } \\
(2013)\end{array}$} \\
\hline & AR & BS & BV & GA & IT & $\mathrm{JO}$ & SF & SJ & \\
\hline & 0 & 0 & 0 & 1,2 & 0,0 & 2,0 & 0,8 & 0 & \\
\hline \multirow{3}{*}{$S$} & \multicolumn{8}{|c|}{ Expectativa de anos de estudo da população } & \multirow{3}{*}{ PNUD } \\
\hline & AR & BS & BV & GA & IT & $\mathrm{JO}$ & SF & SJ & \\
\hline & 9,4 & 9,8 & 9,9 & 10, & 10, & 10, & 10, & 9,84 & \\
\hline I & \multicolumn{8}{|c|}{ Tempo médio habitual de deslocamentos para o } & \multirow{7}{*}{$\begin{array}{l}\text { Feco- } \\
\text { mércio } \\
(2012)\end{array}$} \\
\hline Tempo & $\mathrm{AR}$ & BS & BV & GA & IT & $\mathrm{JO}$ & SF & SJ & \\
\hline$>5$ min. & 15, & 30 , & 24 & 24 & 27 , & 9,1 & 17 & 18,27 & \\
\hline 6-30 min & 40 & 49 , & 60 & 63 & 59 , & 57 & 66 & 63,07 & \\
\hline 0,5- 1 hora & 30 & 16 & 11 & 9,8 & 10 & 27 & 11 & 16,44 & \\
\hline $1-2$ horas & 12 & 3,3 & 2,9 & 2,4 & 2,1 & 5,6 & 3,4 & 1,95 & \\
\hline$<2$ horas & 0,4 & 0 & 0,8 & 0,1 & 0,6 & 0,3 & 0,8 & 0,27 & \\
\hline
\end{tabular}




\begin{tabular}{|c|c|c|c|c|c|c|c|c|c|}
\hline$\%$ total & 100 & 100 & 100 & 100 & 100 & 100 & 100 & 100 & \\
\hline \multirow{3}{*}{ I } & \multicolumn{8}{|c|}{ Número de óbitos infantis por mil nascidos vivos } & \multirow{3}{*}{$\begin{array}{l}\text { PNUD } \\
(2010)\end{array}$} \\
\hline & AR & BS & BV & GA & $\mathrm{IT}$ & $\mathrm{JO}$ & SF & SJ & \\
\hline & 13 & 12 & 11 & 13, & 10, & 9,3 & 11 & 10,5 & \\
\hline \multirow{3}{*}{$\mathrm{R}$} & \multicolumn{8}{|c|}{ Percentual de despesa pública municipal com } & \multirow{3}{*}{$\begin{array}{c}\text { STN } \\
(2013)\end{array}$} \\
\hline & AR & BS & BV & GA & $\mathrm{IT}$ & $\mathrm{JO}$ & SF & SJ & \\
\hline & 10 & 1,5 & 0 & 0,3 & 0 & 0 & 0,2 & 0 & \\
\hline $\mathrm{R}$ & \multicolumn{8}{|c|}{ Número de unidades básicas públicas de atendimento } & \multirow{5}{*}{$\begin{array}{c}\text { Datasus } \\
\text { (2015) }\end{array}$} \\
\hline Unidades & AR & BS & BV & GA & IT & $\mathrm{JO}$ & SF & SJ & \\
\hline Centro & 5 & 3 & 3 & 5 & 5 & 59 & 14 & 4 & \\
\hline Posto & 4 & 0 & 0 & 0 & 0 & 0 & 4 & 0 & \\
\hline P.A. & 0 & 0 & 1 & 1 & 0 & 3 & 1 & 0 & \\
\hline \multirow{3}{*}{$\mathrm{R}$} & \multicolumn{8}{|c|}{ Percentual de despesa pública municipal com saúde no } & \multirow{3}{*}{$\begin{array}{c}\text { STN } \\
(2013)\end{array}$} \\
\hline & AR & BS & BV & GA & $\mathrm{IT}$ & $\mathrm{JO}$ & SF & SJ & \\
\hline & 17 & 21 & 23 & 23 & 21 & 36 & 18 & 21,59 & \\
\hline \multirow{3}{*}{$\mathrm{R}$} & \multicolumn{8}{|c|}{ Percentual de despesa pública municipal com } & \multirow{3}{*}{$\begin{array}{c}\text { STN } \\
(2013)\end{array}$} \\
\hline & AR & BS & BV & GA & IT & $\mathrm{JO}$ & SF & SJ & \\
\hline & 37 & 22 & 35 & 32 & 33, & 24 & 22 & 23,58 & \\
\hline \multirow{3}{*}{$\mathrm{R}$} & & centu & de ir & estir & ento & er ca & a em & Itura no & \multirow{3}{*}{$\begin{array}{c}\text { STN } \\
(2013)\end{array}$} \\
\hline & $\mathrm{A}, 0$ & BS & BV & $\mathrm{GA}$ & IT & JO & SF & SJ & \\
\hline & 25 & 96 & 3,9 & 37 & 20 & 25 & 39 & 1,43 & \\
\hline
\end{tabular}

Araquari (AR); B. Barra do Sul (BS); Barra Velha (BV); Garuva (GA); Itapoá (IT); Joinville (JO); São Francisco do Sul (SF); São João do Itaperiú (SJ).

Conforme observado no quadro 1 , no SIMeC considerou-se como força motriz, a taxa de crescimento da população que influencia diretamente na densidade demográfica, o indicador de pressão. Os dados mostram que Araquari, apresenta maior taxa de crescimento populacional que pode estar associada à forte integração com Joinville e à implantação de novas indústrias no município. Itapoá, por conta do porto inaugurado em 2012, tem a segunda maior estimativa de crescimento da população, o que mostra que os grandes empreendimentos têm atraído imigrantes.

As concentrações populacionais, principalmente nas áreas urbanas, interferem no estado do trânsito local tendo em vista o uso de carros particulares para deslocamentos para o trabalho. Destaca-se, além de Joinville, São João de Itaperiú, com a menor população da região sendo que 23,8 de cada 100 habitantes possuem veículos próprios. A taxa de motorização alta aumenta as concentrações de veículos e por sua vez congestiona vias e aumenta o tempo de deslocamento entre casa e trabalho, impacto que também pode ser influenciado pela oferta de empregos em municípios vizinhos aumentando a distância dos deslocamentos. Estas duas possibilidades fazem com que a maior média de deslocamento em todos os municípios esteja entre 6 min. e 30 min., com destaque novamente para Araquari que apresenta a maior média de pessoas se deslocando entre 30 e 60 min. 
Como resposta para melhorar a mobilidade urbana, destaca-se o investimento em transporte, sendo que Araquari tem mostrado ações de investimentos em transporte correspondendo à $10,59 \%$ das despesas totais do município. Como destaque na construção de ciclovia, vale mencionar Joinville com $125 \mathrm{Km}$ de extensão com metas de ampliação estabelecidas no Plano Municipal de Mobilidade Urbana, sendo este o único a apresentar este dado.

Outro fator relevante para qualidade de vida urbana é a expectativa de anos de estudos da população que pode estar associada ao acesso a melhores empregos e salários, assim como melhor conhecimento dos direitos para exigir maior acesso à instrumentos e serviços urbanos. No que se refere a este indicador, todos apresentam expectativa de estudo entre 9 e 10 anos estando apenas Araquari abaixo da média do Brasil que é de 9,54 anos (PNUD, 2010). Como resposta cabe mencionar que este município em 2013, foi o que empregou $37,52 \%$ da despesa total na educação.

Além do acesso à educação, o investimento em cultura, um indicador de resposta, promove a sensação de pertencimento com a promoção das identidades e memórias locais. Nesse caso, os municípios de Araquari e Garuva apresentam o maior investimento per capta em cultura, seguido de Barra Velha e São Francisco do Sul. Com exceção de Garuva, estes municípios apresentam forte relação com a cultura das comunidades tradicionais pesqueiras, sendo de alta relevância para a identidade local.

Também importante, para a qualidade de vida há o acompanhamento da saúde humana. Destaque para Joinville com o maior número de profissionais da saúde, assim como, o maior número de leitos hospitalares, sendo também o que tem maior percentual da despesa pública total com saúde (36,29\%). Esta realidade faz com que as populações dos municípios vizinhos tenham que se deslocar para Joinville para atendimento médico. Como impacto, com exceção de Joinville, todos os municípios da região apresentam o número de óbitos de crianças com menos de um ano, acima do considerado aceitável pela Organização Mundial de Saúde (10 óbitos). Como resposta, está o uso de Unidades Básicas de Saúde como alternativas, por serem distribuídos para estarem mais próximos à população e permitir atendimentos e exames preventivos, assim como, encaminhamentos para hospitais. Joinville também se destaca com a maior oferta. Ao relacionar o número de leitos com este indicador, percebe-se em que alguns dos municípios da região, os postos de saúde, apresentam-se como única alternativa pública. 
Considerando a dimensão de forma geral, na Região de Joinville, observa-se a necessidade de investimentos em saúde, assim como na mobilidade urbana, sendo o investimento em transporte público e na construção de ciclovias uma necessidade. No que se refere à educação, destaca-se os investimentos para melhorar os anos de escolaridade da população.

\section{Infraestrutura}

A qualidade e disponibilidade do saneamento básico, estradas e tecnologias da informação, entre outros, melhora os níveis de vida com o aumento da produtividade e competitividade dos sistemas produtivos (UN-HABITAT, 2012). Assim, a infraestrutura pode funcionar como um catalisador para o crescimento econômico e transformação social (WON; SEA; SAEHOON, 2015; FRISCHTAK, 2013) servindo ao duplo propósito de atrair os investimentos privados e de melhorar o desempenho do setor público (FRISCHTAK, 2013). No quadro 2 são apresentados os indicadores considerados nessa dimensão.

Quadro 2- Região de Joinville e a dimensão da Infraestrutura

\begin{tabular}{|c|c|c|c|c|c|c|c|c|c|}
\hline DPSIR & \multicolumn{8}{|c|}{ Aplicação dos indicadores } & Fonte \\
\hline \multirow{3}{*}{ D } & \multicolumn{8}{|c|}{ Área urbanizada em $\mathrm{Km}^{2}$} & \multirow{3}{*}{$\begin{array}{l}\text { Miranda; Gomes; } \\
\text { Guimarães } \\
(2005)\end{array}$} \\
\hline & AR & BS & BV & $\mathrm{GA}$ & IT & $\mathrm{JO}$ & SF & SJ & \\
\hline & 4,4 & 1,9 & 3,2 & 2,27 & 8,6 & 114 & 5,694 & 0,368 & \\
\hline \multirow{3}{*}{$P$} & \multicolumn{8}{|c|}{ Taxa de urbanização } & \multirow{3}{*}{$\begin{array}{l}\text { Portal } \\
\text { ODM } \\
(2015)\end{array}$} \\
\hline & AR & BS & BV & GA & IT & $\mathrm{JO}$ & SF & SJ & \\
\hline & 94 & 95, & 95, & 77,5 & 96 & 96,6 & 92,87 & 56,04 & \\
\hline \multirow{3}{*}{$\mathrm{P}$} & \multicolumn{8}{|c|}{ Demanda urbana de água em litros por segundo } & \multirow{3}{*}{$\begin{array}{c}\text { ANA } \\
(2015)\end{array}$} \\
\hline & AR & BS & BV & GA & IT & JO & SF & SJ & \\
\hline & 53 & 23 & 55 & 33 & 33 & 1656 & 113 & 5 & \\
\hline \multirow{3}{*}{ S } & \multicolumn{8}{|c|}{$\begin{array}{l}\text { Percentual de domicílios com acesso à rede de esgoto ou } \\
\text { fossa séptica }\end{array}$} & \multirow{3}{*}{$\begin{array}{l}\text { IBGE } \\
(2010)\end{array}$} \\
\hline & AR & BS & BV & GA & $\mathrm{IT}$ & $\mathrm{JO}$ & SF & SJ & \\
\hline & 64, & 83 , & 88 , & 58,1 & 90, & 75,7 & 76,56 & 56,3 & \\
\hline \multirow{3}{*}{$\mathrm{s}$} & \multicolumn{8}{|c|}{ Percentual de Domicílios ligados a rede de esgoto ou } & \multirow{3}{*}{$\begin{array}{l}\text { IBGE } \\
(2010)\end{array}$} \\
\hline & AR & BS & BV & GA & $\mathrm{IT}$ & $\mathrm{JO}$ & SF & SJ & \\
\hline & 35 & 9,2 & 10, & 20,9 & 1 & 34,8 & 11 & 2,75 & \\
\hline \multirow{3}{*}{$\mathrm{s}$} & \multicolumn{8}{|c|}{$\begin{array}{c}\text { Percentual da população urbana residente em domicílios } \\
\text { com coleta de lixo }\end{array}$} & \multirow{3}{*}{$\begin{array}{l}\text { PNUD } \\
(2010)\end{array}$} \\
\hline & AR & BS & BV & GA & IT & JO & SF & SJ & \\
\hline & 98, & 99, & 98, & 99,0 & 99, & 99,9 & 99,09 & 98,9 & \\
\hline \multirow{3}{*}{$S$} & $\% 0$ & pes & $\operatorname{ass} r$ & $\begin{array}{l}\text { ident } \epsilon \\
\text { de } \epsilon\end{array}$ & $\begin{array}{ll}\text { em c } \\
\text { eraia }\end{array}$ & $\begin{array}{l}\text { micílio } \\
\text { létrica }\end{array}$ & com for & cimento & \multirow{3}{*}{$\begin{array}{l}\text { PNUD } \\
(2010)\end{array}$} \\
\hline & AR & BS & BV & $\mathrm{GA}$ & IT & $\mathrm{JO}$ & SF & SJ & \\
\hline & 99 & 99 , & 99 , & 99,9 & 99 & 99,9 & 99,22 & 99,79 & \\
\hline \multirow{3}{*}{$S$} & & Perc & $\overline{\text { ntua }}$ & don & sílios & $\mathrm{om} \mathrm{ac \epsilon}$ & so à int & & \multirow{3}{*}{$\begin{array}{l}\text { IBGE } \\
(2010)\end{array}$} \\
\hline & AR & BS & BV & $\mathrm{GA}$ & IT & $\mathrm{JO}$ & SF & SJ & \\
\hline & 13 & 16 & 27, & 39,3 & 28 & 47,5 & 32,47 & 12,75 & \\
\hline
\end{tabular}




\begin{tabular}{|c|c|c|c|c|c|c|c|c|c|}
\hline \multirow{3}{*}{ S } & \multicolumn{8}{|c|}{$\begin{array}{l}\text { Percentual da população residente em domicílios com } \\
\text { água encanada }\end{array}$} & \multirow{3}{*}{$\begin{array}{l}\text { PNUD } \\
(2010)\end{array}$} \\
\hline & AR & BS & BV & GA & IT & JO & SF & SJ & \\
\hline & 96, & 98,9 & 98,6 & 85,1 & 97,3 & 98,1 & 96,7 & 96,7 & \\
\hline \multirow{3}{*}{ I } & \multicolumn{8}{|c|}{ Geração de resíduos sólidos (ton./dia) } & \multirow{3}{*}{$\begin{array}{l}\text { SDC } \\
(\mathrm{SC}) \\
(2012)\end{array}$} \\
\hline & AR & BS & BV & GA & IT & $\mathrm{JO}$ & SF & SJ & \\
\hline & 12,4 & 3,37 & 11,1 & 7,38 & 7,38 & 412,23 & 29,76 & 1,37 & \\
\hline $\mathrm{I}$ & \multicolumn{8}{|c|}{$\begin{array}{l}\text { Número de ocorrências de desastres naturais por período } \\
\qquad(2005-2010)\end{array}$} & \multirow{4}{*}{$\begin{array}{l}\text { Atlas dos } \\
\text { desastres } \\
(2012)\end{array}$} \\
\hline Tipo & AR & BS & BV & GA & IT & $\mathrm{JO}$ & SF & SJ & \\
\hline Inundaçã & 0 & 4 & 3 & 0 & 0 & 7 & 0 & 0 & \\
\hline Vendaval & 0 & 0 & 2 & 1 & 0 & 2 & 0 & 0 & \\
\hline $\mathrm{I}$ & \multicolumn{8}{|c|}{$\begin{array}{l}\text { Nível de erosão costeira } \\
\text { am encontrados dados na região }\end{array}$} & \\
\hline $\mathrm{R}$ & \multicolumn{8}{|c|}{$\begin{array}{c}\text { Presença de emissários submarinos para esgotos } \\
\text { domésticos e efluentes } \\
\text { Não há emissários na região. }\end{array}$} & \\
\hline $\mathrm{R}$ & \multicolumn{8}{|c|}{$\begin{array}{l}\text { Destinação final dos resíduos sólidos urbanos } \\
\text { odos os municípios destinam os resíduos à aterros } \\
\text { sanitários }\end{array}$} & MP/SC (2012) \\
\hline $\mathrm{R}$ & \multirow{2}{*}{\multicolumn{8}{|c|}{$\begin{array}{l}\text { Linha de costa 'protegida' por estruturas artificiais e molhes } \\
\qquad(\% / \text { ano) } \\
\text { Não foram encontrados dados para a região. }\end{array}$}} & \\
\hline & & & & & & & & & \\
\hline $\mathrm{R}$ & \multicolumn{8}{|c|}{$\begin{array}{c}\text { Número de praias e marinas com certificação ambiental } \\
\text { Não há praias ou marinas certificadas na região. }\end{array}$} & $\begin{array}{l}\text { Bandeira Azul } \\
(2015))\end{array}$ \\
\hline \multirow{3}{*}{$\mathrm{R}$} & \multicolumn{8}{|c|}{ Percentual das despesas municipais com urbanismo/ano } & \multirow{3}{*}{$\begin{array}{l}\text { STN } \\
(2013)\end{array}$} \\
\hline & AR & BS & BV & GA & IT & $\mathrm{JO}$ & SF & SJ & \\
\hline & 0 & 0,9 & 11 & 8,43 & 12 & 9,62 & 9,65 & 23,02 & \\
\hline
\end{tabular}

Araquari (AR); B. Barra do Sul (BS); Barra Velha (BV); Garuva (GA); Itapoá (IT); Joinville (JO); São Francisco do Sul (SF); São João do Itaperiú (SJ).

Conforme o quadro 2, como força motriz desta dimensão está a extensão das áreas urbanizadas. Neste caso, Joinville apresenta uma área urbana muito maior que os outros municípios, no entanto, quando trata-se do percentual da população morando em áreas urbanas, a taxa de urbanização de Garuva e São João do Itaperiú mostram uma população rural considerável, oposto dos outros municípios com taxa de urbanização acima de $90 \%$.

A maior concentração de pessoas e a extensão da área urbana aumentam a necessidade de serviços. Exemplo é Joinville com a maior demanda por abastecimento de água. Nos indicadores de estado, destaca-se o percentual baixo de domicílios ligados a rede de esgoto ou pluvial o que tende a impactar negativamente na qualidade ambiental das bacias hidrográficas da região (indicador da sustentabilidade ambiental). A alta geração de resíduos em Joinville mostra um alto nível de consumo, como resposta, a infraestrutura deve permitir a destinação 
adequada do lixo em aterros sanitários, sendo que todos os municípios encaminham esse material para aterros sanitários à distâncias que podem chegar a aproximadamente $180 \mathrm{Km}$, caso de Itapoá.

No que se refere ao acesso à Tecnologia de Informação e Comunicação, todos os municípios da região, não atingem $50 \%$ dos domicílios com acesso à internet. Outro problema enfrentado pela região é a população vulnerável e o número de enxurradas e inundações. A erosão costeira também é perceptível em praias da região a exemplo dos municípios de Balneário Barra do Sul, Barra Velha, Itapoá e São Francisco do Sul, no entanto, a região não apresenta dados disponíveis do nível de erosão e das medidas de contenção. Já para as despesas municipais com urbanismo, destacam-se os gastos de São João de Itaperiú, o município menos urbanizado da Região.

No contexto global, os indicadores apontam a necessidade de melhorar os investimentos principalmente com o tratamento adequado de esgoto o que impacta diretamente na qualidade do ambiente costeiro. Essa dimensão apresenta indicadores abrangentes para todas as metrópoles e específicos para as áreas costeiras. No que se refere aos específicos, a região carece de pensar a realidade costeira e desenvolver programas para estudar e divulgar informações.

\section{Produtividade}

Uma análise coerente entre a questão econômica e a sustentabilidade requer a integração de diferentes temáticas que incluem a própria viabilidade econômica junto aos limites ecossistêmicos e à justiça social. Para tanto deve-se fazer uso racional das infraestruturas como, por exemplo, nas estratégias de logística urbana, arranjos comerciais, consumo e oferta de emprego com menor impacto ambiental (OLIVEIRA; CORREIA, 2014; ERKIP; KIZILGUN; AKINCI, 2014; ZHANG; LIU; FATH, 2014). Assim, a produtividade neste estudo é entendida como a capacidade dos setores produtivos das cidades de aproveitarem oportunidades para melhorar 0 desempenho econômico com menor impacto ambiental (UN-HABITAT, 2012). O quadro 3 apresenta os indicadores relacionados a dimensão produtividade.

Quadro 3- Região de Joinville e a dimensão da Produtividade

\begin{tabular}{|c|c|c|c|}
\hline DPSIR & \multicolumn{2}{|c|}{ Aplicação dos indicadores } & Font \\
\hline \multirow{3}{*}{} & \multicolumn{2}{|c}{ PIB a preços correntes/ano (por mil reais) } & \multirow{2}{*}{ Valores } \\
\cline { 2 - 3 } & Muni & 1.002 .590 & \\
\cline { 2 - 3 } & AR & 145.369 & \\
\cline { 2 - 3 } & BS & \\
\hline
\end{tabular}


ADÃO, POLETTE

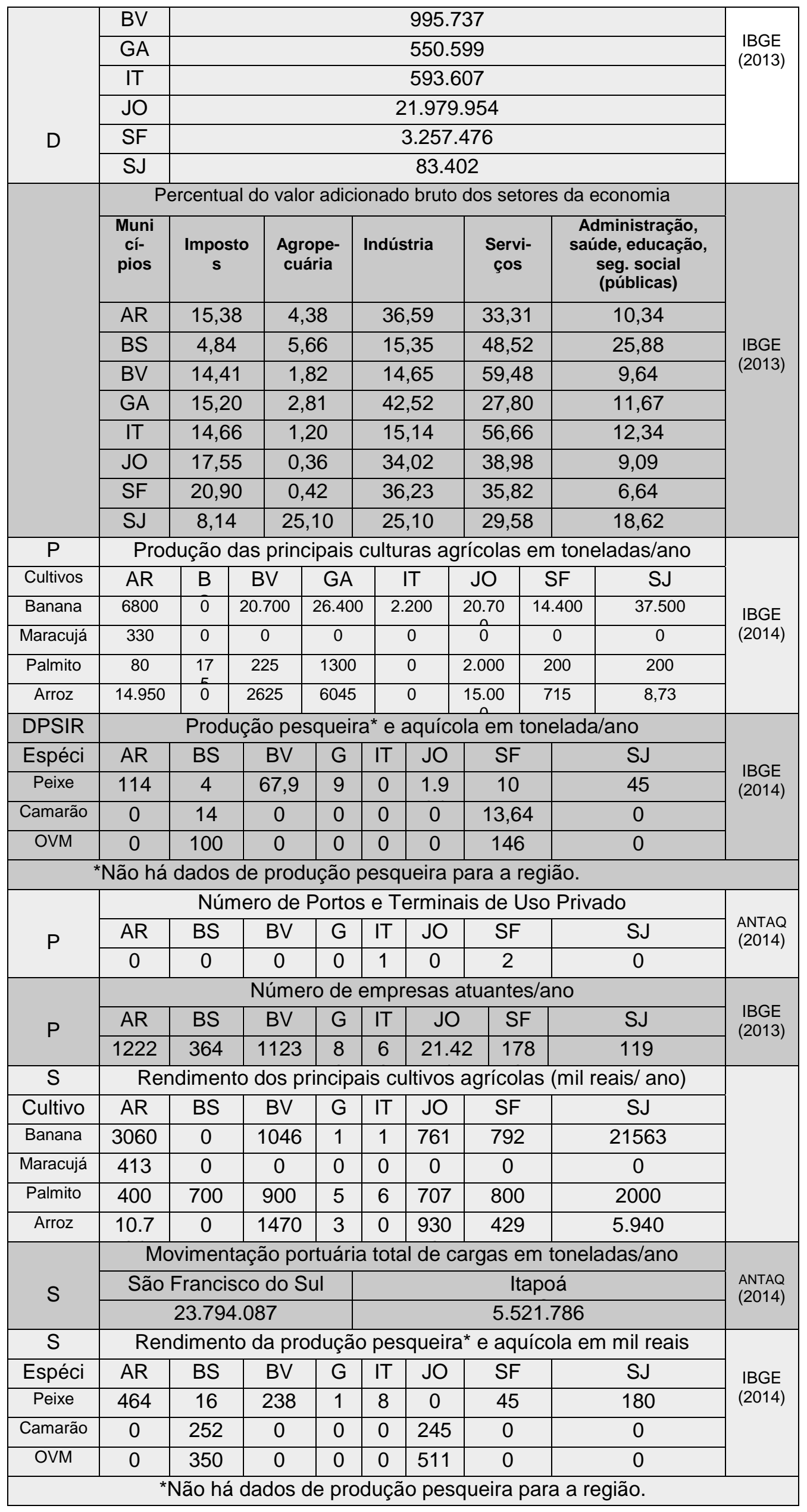




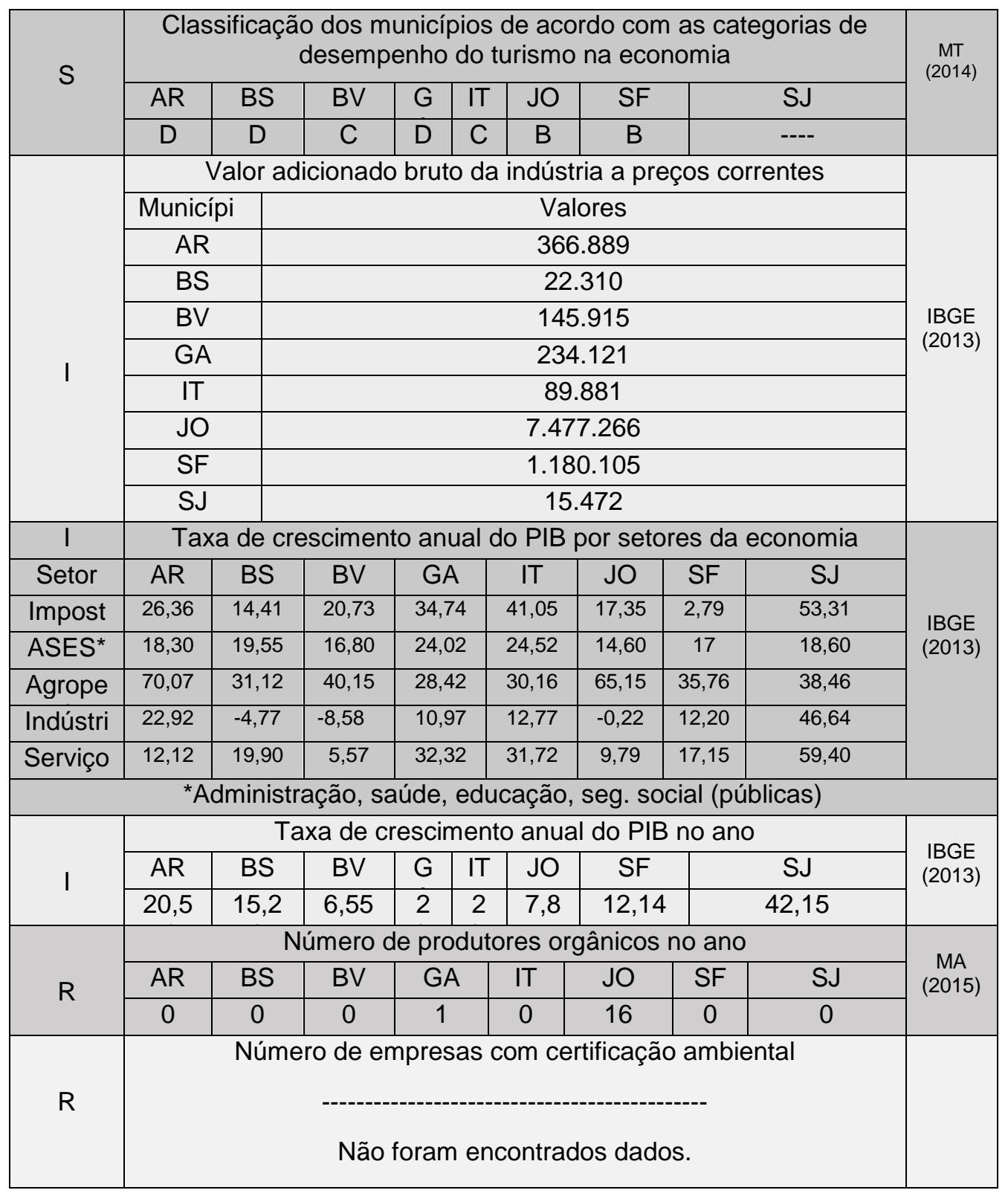

Araquari (AR); B. Barra do Sul (BS); Barra Velha (BV); Garuva (GA); Itapoá (IT); Joinville (JO); São Francisco do Sul (SF); São João do Itaperiú (SJ).

Como observado no quadro 3, foi considerado como força motriz o Produto Interno Bruto, total e por setores da economia. Como pressão, evidenciou-se a produção nos diferentes setores. Para identificar o potencial turístico, usou-se a classificação do Ministério do Turismo, considera as variáveis: emprego formal, estrutura hoteleira e estimativa de turistas. Nessa classificação os municípios de Joinville, São Francisco, classificados como B, e Barra Velha e Itapoá, classificados como $\mathrm{C}$, os três últimos com o turismo relacionado ao uso das praias da região. $\mathrm{Na}$ produção pesqueira e aquícola, a aquicultura se mostra como a alternativa para comunidades tradicionais e a produção pesqueira não pode ser dimensionada por não haver local específico para o desembarque de pescado na região. 
A existência de dois portos e um terminal marítimo influencia diretamente nos setores econômicos, na arrecadação de impostos sobre serviços, geração de emprego e de uso das empresas instaladas na região. Nos indicadores de estado, considerou-se o rendimento dos setores econômicos. Apesar de se caracterizar como uma região industrial, o setor terciário é o que apresenta maior crescimento. Como respostas, a região carece de um sistema de divulgação de empresas com certificações ambientais a exemplo dos produtores orgânicos registrados no Ministério da Agricultura.

Em uma análise global, essa dimensão, além da industrialização associada às instalações portuárias, aquicultura e pesca; o setor de serviços, também associado à essas atividades, apresentam potencialidades por conta dos atrativos turísticos litorâneos.

\section{Sustentabilidade Ambiental}

As cidades ambientalmente sustentáveis são menos poluidoras, oferecem opções de infraestrutura menos impactante ambientalmente e oferece melhores oportunidades para a sociedade e são também mais resilientes aos fenômenos naturais (UN-HABITAT, 2012; FOLADORI, 1999; SANTOS, 2014). No quadro 4 apresenta-se os indicadores que foram considerados nessa dimensão.

Quadro 4- Região de Joinville e a Sustentabilidade Ambiental

\begin{tabular}{|c|c|c|c|c|c|c|c|c|c|}
\hline DPSIR & \multicolumn{8}{|c|}{ Aplicação dos indicadores } & Fonte \\
\hline \multirow{3}{*}{ D } & \multicolumn{8}{|c|}{ Área urbanizada do município em $\mathrm{Km}^{2}$} & \multirow{3}{*}{$\begin{array}{c}\text { Miranda; } \\
\text { Gomes; } \\
\text { Guimarães } \\
(2005)\end{array}$} \\
\hline & AR & BS & BV & GA & IT & $\mathrm{JO}$ & SF & SJ & \\
\hline & 4,433 & 1,9317 & $\begin{array}{c}3,262 \\
2\end{array}$ & 2,277 & 8,64 & $\begin{array}{c}114,49 \\
6\end{array}$ & 5,694 & 0,368 & \\
\hline \multirow{3}{*}{$\mathrm{P}$} & \multicolumn{8}{|c|}{ Percentual de áreas naturais protegidas em relação à área total } & \multirow{3}{*}{$\begin{array}{l}\text { IBGE } \\
\text { SNUC } \\
(2015)\end{array}$} \\
\hline & AR & BS & BV & GA & IT & $\mathrm{JO}$ & SF & SJ & \\
\hline & 0 & 0 & 0 & 0 & 0,049 & 0,0409 & 13,38 & 0 & \\
\hline \multirow[t]{2}{*}{$\mathrm{P}$} & \multicolumn{8}{|c|}{ Percentual de áreas de manguezais em relação a área total } & \multirow{2}{*}{$\begin{array}{c}\text { GERCO } \\
(2010)\end{array}$} \\
\hline & \multicolumn{8}{|c|}{ Margens da Baia da Babitonga ( $2 \%$ da área total) } & \\
\hline \multirow[b]{2}{*}{ S } & \multicolumn{8}{|c|}{ Área verde por habitantes/ano } & \multirow{2}{*}{$\begin{array}{c}\text { Prefeituras } \\
(2015)\end{array}$} \\
\hline & \multicolumn{8}{|c|}{ Somente Joinville apresenta esse dado (2014=1368 $\mathrm{m}^{2} /$ habitante) } & \\
\hline \multirow[t]{2}{*}{ S } & \multicolumn{8}{|c|}{ Índice da Qualidade da Água (IQA) por pontos das Bacias } & \multirow{7}{*}{$\begin{array}{c}\text { SEMA/ } \\
\text { Joinville } \\
(2014)\end{array}$} \\
\hline & Cubatão & Cach & & Quiriri & Palmital & Piraí & Vertent & Leste & \\
\hline Ótimo & 1 & ( & & 1 & 0 & 1 & ( & & \\
\hline Bom & 6 & ( & & 0 & 3 & 5 & ( & & \\
\hline Regular & 0 & 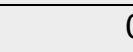 & & 0 & 0 & 1 & ( & & \\
\hline Ruim & 0 & 3 & & 0 & 0 & 0 & 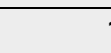 & & \\
\hline \multirow[t]{2}{*}{ Péssimo } & 1 & 2 & & 0 & 0 & 2 & 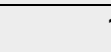 & & \\
\hline & \multicolumn{8}{|c|}{ Índice de Qualidade do Ar (IQAR) } & \\
\hline
\end{tabular}




\begin{tabular}{|c|c|c|c|c|c|c|c|c|c|}
\hline $\mathrm{S}$ & \multicolumn{8}{|c|}{ Santa Catarina não possui sistema de monitoramento do ar. } & \\
\hline S & \multicolumn{8}{|c|}{$\begin{array}{l}\text { Número de espécies da avifauna em área urbana } \\
\text {-------- } \\
\text { Foram observadas } 241 \text { espécies de aves. (local: Morro do Finder, Boa } \\
\text { Vista e Parque Caieira) }\end{array}$} & $\begin{array}{l}\text { Grose } \\
(2013)\end{array}$ \\
\hline \multirow{3}{*}{$S$} & \multicolumn{8}{|c|}{ Condições de balneabilidade/ano (medidas próprias/m. impróprias) } & \multirow{3}{*}{$\begin{array}{c}\text { FATMA } \\
(2015)\end{array}$} \\
\hline & \multirow{2}{*}{\multicolumn{3}{|c|}{ BS }} & BV & IT & $\mathrm{JO}$ & \multicolumn{2}{|c|}{ SF } & \\
\hline & & & & $48 / 5$ & $75 / 1$ & $13 / 9$ & $1 / 41(3 r$ & thos) & \\
\hline 1 & \multicolumn{8}{|c|}{$\begin{array}{l}\text { № total de acidentes ambientais com efeitos em cursos d'água que } \\
\text { ocasionaram mortandade de peixes } \\
\text { De } 2009 \text { à } 2012 \text { Joinville apresentou } 8 \text { casos ( } 5^{\circ} \text { colocado no país) }\end{array}$} & $\begin{array}{l}\text { ANA } \\
(2013)\end{array}$ \\
\hline $\mathrm{I}$ & \multicolumn{8}{|c|}{$\begin{array}{c}\text { Número de ocorrências de algas nocivas e microtoxinas } \\
\text { Região livre de algas nocivas e microtoxinas }\end{array}$} & $\begin{array}{l}\text { CIDASC } \\
(2015)\end{array}$ \\
\hline DPSIR=I & \multicolumn{8}{|c|}{ Casos de doenças infecto-parasitárias relacionadas à água relevantes } & \multirow{6}{*}{$\begin{array}{l}\text { Datasus } \\
(2015)\end{array}$} \\
\hline Doenças & AR & BS & BV & GA & IT & $\mathrm{JO}$ & SF & SJ & \\
\hline Filarióse & 0 & 0 & 0 & 0 & 0 & 0,2 & 0 & 0 & \\
\hline Amebíase & 0 & 0 & 0 & 0 & 0 & 0,2 & 0 & 0 & \\
\hline Leptospirose & 3,7 & 11,4 & 0 & 0 & 12,8 & 6,5 & 9,1 & 28,8 & \\
\hline Dengue & 0 & 0 & 0 & 0 & 6,4 & 2,3 & 2,3 & 0 & \\
\hline \multirow{3}{*}{$R$} & \multicolumn{8}{|c|}{ Percentual de despesas com gestão ambiental dos municípios } & \multirow{3}{*}{$\begin{array}{l}\text { STN } \\
(2013)\end{array}$} \\
\hline & AR & BS & BV & GA & IT & $\mathrm{JO}$ & SF & SJ & \\
\hline & 1,30 & 0 & 0,52 & 2,10 & 0,28 & 1,24 & 0,49 & 0,06 & \\
\hline \multirow{3}{*}{$\mathrm{R}$} & \multicolumn{8}{|c|}{ Áreas de Unidades de Conservação $\left(\mathrm{km}^{2}\right)$} & \multirow{3}{*}{$\begin{array}{l}\text { MMA } \\
(2015)\end{array}$} \\
\hline & & & & Joinvi & & São & ancisco & Sul & \\
\hline & & & & 461,1 & & & 66,751 & & \\
\hline \multirow{3}{*}{$\mathrm{R}$} & \multicolumn{8}{|c|}{$\begin{array}{l}\text { Municípios costeiros da região com Projeto de Gestão Integrada (PGI) } \\
\text { do Projeto Orla }\end{array}$} & \multirow[t]{3}{*}{$\begin{array}{c}\text { MMA } \\
(2016)\end{array}$} \\
\hline & AR & BS & BV & $\mathrm{GA}$ & IT & $\mathrm{JO}$ & SF & SJ & \\
\hline & Não & Não & Não & Não & Sim & Não & Não & ---- & \\
\hline
\end{tabular}

Araquari (AR); B. Barra do Sul (BS); Barra Velha (BV); Garuva (GA); Itapoá (IT); Joinville (JO); São Francisco do Sul (SF); São João do Itaperiú (SJ).

Como percebido no quadro 4, na dimensão da sustentabilidade ambiental também se considerou como força motriz a área urbanizada que consiste na relação da área urbana com a área total. Como indicadores de pressão foram consideradas as relações entre áreas protegidas e de manguezais com a área total. Observa-se que São Francisco do Sul apresenta percentual expressivo de $13,38 \%$ de áreas protegidas. Já as principais áreas de manguezais cobrem $2 \%$ da área total estando diretamente relacionadas ao ecossistema da Baia da Babitonga que margeia seis municípios da região.

No que se refere ao estado, a região ainda apresenta um número significativo de avifauna nas áreas urbanas em que foi avistado $40 \%$ das espécies encontradas em Santa Catarina. Quanto ao índice de qualidade da água (IQA), atenção deve ser dada principalmente à Bacia Hidrográfica do Rio Cachoeira, que atravessa toda região central de Joinville. Como limitação, o estado de Santa Catarina ainda não possui um sistema para 
monitoramento representando um atraso para as estratégias de gestão ambiental. Quanto a balneabilidade, a região possui 20 pontos com análises realizadas durante o verão. Como resultado, somente três praias de São Francisco do Sul apresentaram, em 24 análises, todas as classificações como próprias para o banho. Já no que se refere a divulgação de área verde por habitantes, somente Joinville disponibiliza informações estando muito acima do mínimo de $12 \mathrm{~m}^{2}$ indicado pela OMS.

Quanto aos impactos, a região se apresenta livre de algas nocivas e microtoxinas. E a leptospirose, em 2012, representou o maior número de casos de doenças infectoparasitárias relacionadas à água, fato diretamente relacionado com as chuvas e enchentes ocorridas na região. Já Joinville, entre os anos de 2009 e 2012 apresentou oito casos de acidentes com mortandade de peixes, sendo o quinto município do Brasil com maior número de casos. Dos indicadores de respostas, somente Itapoá, Joinville e São Francisco do Sul apresentam áreas protegidas. Somente Itapoá possui Plano de Gerenciamento Costeiro concluído e quanto aos gastos municipais com gestão ambiental, Garuva apresentou o melhor resultado aplicando 2,1\% da sua despesa total em 2013, já Balneário Barra do Sul não realizou gastos com gestão ambiental.

Nessa dimensão, aspectos dos ambientes costeiros são associados a relação espacial entre área urbana e áreas naturais. Pela sua característica, a mesma pode ser evidenciada nas relações entre os ambientes naturais e urbanos, seus impactos e as ações e investimentos para proteção do ambiente natural. No caso da Região de Joinville, deve-se desenvolver ações com foco em gestão costeira por meio dos Projetos de Gestão Integrada e definir estratégias para melhoria da qualidade dos rios e praias oceânicas. A falta de um sistema de monitoramento da qualidade do ar impossibilita saber a real magnitude das emissões de poluentes na atmosfera.

\section{Inclusão e Equidade Social}

A inclusão e a equidade social estão associadas à liberdade de acessos legitimados pelos instrumentos de participação social nas decisões políticas e na universalização dos direitos e melhoria das condições socioeconômicas (SEN, 2000; SILVA, 2014; BARROCO, 2014; DEDECCA; TROVÃO; SOUZA, 2014; UNHABITAT, 2012). No quadro 5 apresenta-se o resultado da aplicação dos indicadores.

Quadro 5- Região de Joinville e a Inclusão e Equidade Social

\begin{tabular}{|c|c|c|c|c|c|c|c|c|c|}
\hline DPSIR & \multicolumn{7}{|c|}{ Aplicação dos indicadores } & Fonte \\
\hline \multirow{3}{*}{$\mathrm{D}$} & \multicolumn{7}{|c|}{ IBGE } \\
\cline { 2 - 7 } & AR & BS & BV & GA & IT & JO & SF & SJ & (2015) \\
\cline { 2 - 7 } & 4,6 & 2,6 & 2,8 & 2,1 & 3,5 & 1,3 & 2,2 & 0,7 & \\
\hline & \multicolumn{8}{|c|}{ Densidade demográfica } & IBGE \\
\hline
\end{tabular}




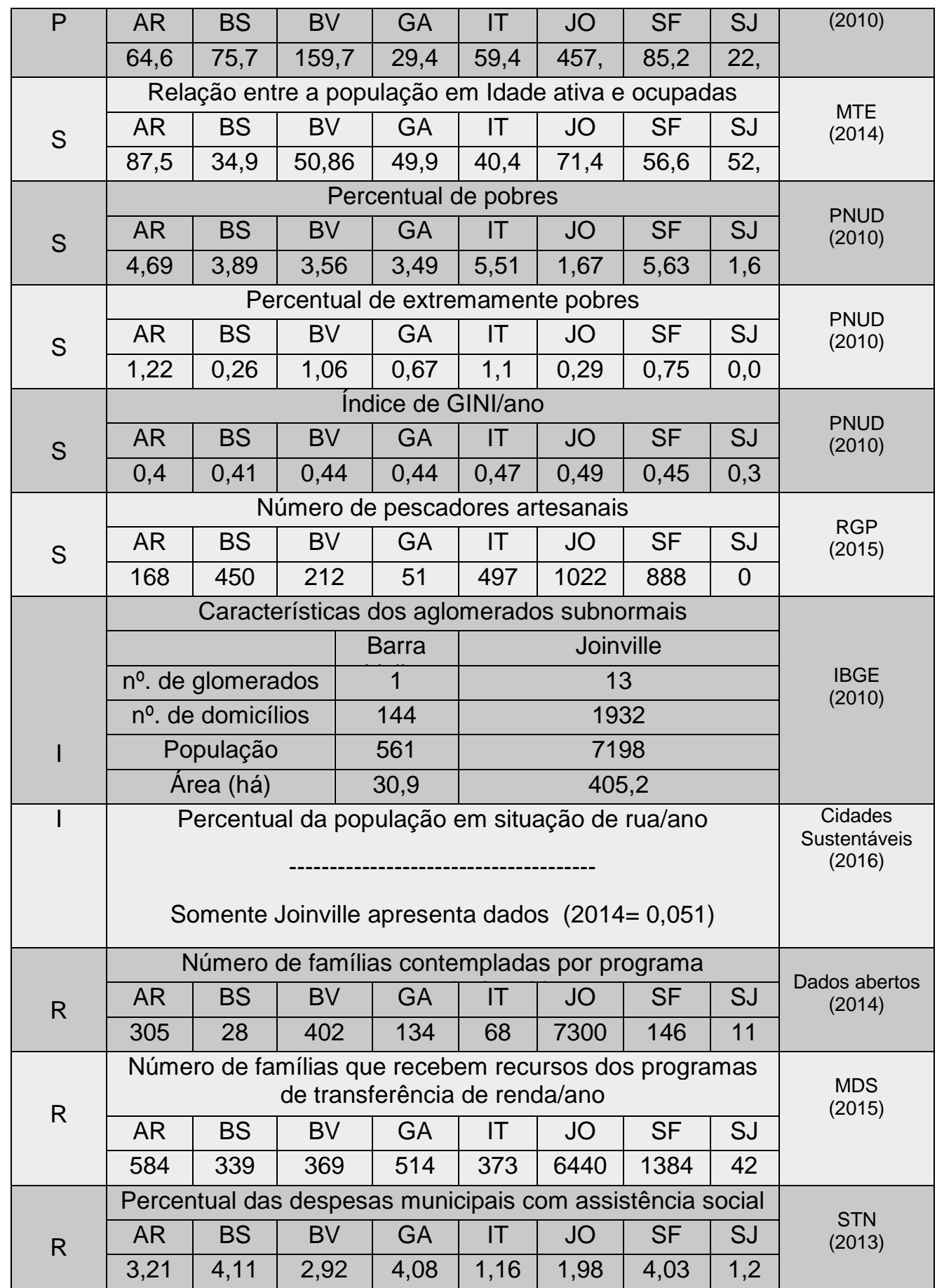

Araquari (AR); B. Barra do Sul (BS); Barra Velha (BV); Garuva (GA); Itapoá (IT); Joinville (JO); São Francisco do Sul (SF); São João do Itaperiú (SJ).

Conforme o quadro 5, para esta dimensão são considerados os mesmos indicadores de força motriz e pressão da dimensão de qualidade de vida. Quanto aos indicadores de estado, as maiores oportunidades de emprego estão nos municípios com maior estrutura urbana. $\mathrm{Na}$ relação entre a população economicamente ativa e a efetivamente empregada, Araquari com os novos empreendimentos ocupa $87,56 \%$ e Joinville possui 71 , $48 \%$ da sua população economicamente ativa ocupada. $O$ baixo percentual de ocupações dos outros 
municípios está relacionado ao fato de muitas pessoas trabalharem, principalmente nos dois com maior número de ocupações. Como resultado, a região como um todo possui $68,85 \%$ da população em idade economicamente ativa ocupada.

O número de pescadores artesanais também permite a análise da relação dos municípios com as comunidades tradicionais que dependem da qualidade do ambiente natural para a geração de renda. Destaque para Joinville com número significativo de pescadores artesanais na Baia da Babitonga e São Francisco do Sul, município de tradição pesqueira.

De maneira antagônica, Araquari que apresenta a maior proporção de pessoas ocupadas, apresenta um número significativo de pobres e extremamente pobres. Sendo Joinville, detentor do segundo maior GINI da região, portanto de maior desigualdade, assim como também o maior número de moradias em aglomerados subnormais. Joinville é o único município da região que apresenta dados de moradores de rua que normalmente se concentram nos maiores centros urbanos. No que se refere às despesas municipais com assistência social, destaca-se o município de Barra do Sul, com $4,11 \%$ dos seus gastos associados aos problemas sociais.

As informações de oferta de empregos associadas aos dados de desigualdade mostram, que não se pode analisar a condição social somente na perspectiva de mercado. Como resposta, São Francisco do Sul, com alta proporção de pobres e extremamente pobres, possui o maior percentual e pessoas atendidas por programas de transferência de renda. Quanto à população atendida pelo "Programa Minha Casa, Minha Vida" (MCMV), destaque para Joinville que também apresenta um número significativo de aglomerados subnormais.

Ao expor os dados desta e das outras dimensões, percebe-se que as possibilidades de análise não se esgotam neste artigo. As relações entre os indicadores, assim como entre as dimensões, devem ser evidenciadas para monitorar e desenvolver ações para o desenvolvimento territorial regional. Da mesma forma, ao associar indicadores específicos de costa com os necessários para o entendimento de ambientes urbanos, o SIMeC se mostrou como uma ferramenta que permite a possibilidade de análise além da apresentada neste artigo.

\section{Considerações Finais}

Ao aplicar o sistema de indicadores de qualidade ambiental urbana na metrópole costeira de Joinville foi possível verificar relações entre o crescimento 
econômico e urbano da região e seus impactos na sociedade e no meio natural. Indicadores considerados de alta relevância para o entendimento e monitoramento dessa realidade ainda não estão disponíveis, como no caso da qualidade do ar e da extensão de erosão costeira, ou não cobrem toda a realidade estudada, como o IQA que ainda não é monitorado em todas as bacias da região. Saber dessa realidade não invalida esses indicadores. Pelo contrário, mostra a necessidade de ampliar ações de coleta de dados e de monitoramento.

Das principais ameaças observadas, além de não haver cobertura total ou parcial de dados para determinados indicadores, está a relação entre a periodicidade da coleta e o tempo de publicação com a validade dessas informações. Em contrapartida, diante da evolução das tecnologias da informação e comunicação, reduziu-se o tempo de divulgação de alguns dados por conta de cadastros informatizados que se tornam banco de dados acessíveis, sendo exemplos, as informações referentes à demanda por água ou sobre as áreas protegidas.

Considerando as questões apresentadas, o sistema se apresentou como uma relevante ferramenta para entender a realidade da uma aglomeração urbana ainda em processo de urbanização e industrialização nas zonas costeiras. Destarte, a aplicação desse sistema apresentou-se válida para a promoção da discussão referente à gestão costeira em ambientes urbanos nas premissas da qualidade ambiental urbana.

\section{REFERÊNCIAS}

ADÃO, Nilton Manoel Lacerda; POLETTE, Marcus. Aplicação do Sistema de Indicadores de Qualidade Ambiental Urbana para Metrópoles Costeiras (SIMeC) nas Regiões Metropolitanas do Recife (PE), Rio de Janeiro (RJ) e na Região de Joinville (SC). Ra'ega O Espaço Geográfico em Análise, v. 44, p. 104-123, maio 2018.

ADÃO, Nilton Manoel Lacerda; POLETTE, Marcus. Sistema de Indicadores de Qualidade Ambiental Urbana para Metrópoles Costeiras (SIMeC): uma proposta de instrumento de análise territorial. Revista Brasileira de Estudos Urbanos e Regionais, v. 18, n. 2, 2016.

BARROCO, Maria Lucia S.. Reflexões sobre liberdade e (in)tolerância. Serv. Soc. Soc. [online]. 2014, n.119, pp. 468-481.

BEROYA-EITNER, Mary Antonette. Ecological vulnerability indicators. Ecological Indicators. 60 (2016) 329-334.

COLL, M. Ecological indicators to capture the effects of fishing on biodiversityand conservation status of marine ecosystems. Ecologicallndicators. 60, 2016, pp. 947-962 
DEDECCA, Claudio Salvadori; TROVAO, Cassiano José Bezerra Marques; SOUZA, Leonardo Flauzinode. Desenvolvimento e equidade: Desafios do crescimento brasileiro. Novos estud. - CEBRAP [online]. 2014, n.98, pp. 23-41.

ERKIP, F., KIZILGUN, Ö.; AKINCI, G. M. Retailers' resilience strategies and their impacts on urban spaces in Turkey. Cities, 36, 112-120, 2014.

FENERI, A. M.; VAGIONA, D.; KARANIKOLAS, N. Measuring quality of life (QOL) in urban enviroment: an integrated approach. CEST2013 Athens, Greece.

FOLADORI, Guillermo. Sustentabilidad ambiental y contradicciones sociales. Ambient. soc. [online], n.5, 1999. pp. 19-34.

FRISCHTAK, Cláudio. Infraestrutura e desenvolvimento no Brasil. In: VELOSO, F. et al. (Orgs.). Desenvolvimento econômico: uma perspectiva brasileira. Rio de Janeiro: Elsevier/Campus, 2013.

GROSE, Alexandre Venson. Avifauna em três unidades de conservação urbanas no município de Joinville, Santa Catarina. Atualidades Ornitológicas, № 175 Setembro/Outubro 2013. Pp. 48-57.

KAMP, Van I.; et. al. Urban environmental quality and human well-being Towards a conceptual framework and demarcation of concepts; a literature study.Landscape and Urban Planning.65 (2003) 5-18.

LAGO, Luciana Corrêa do. Desigualdades e segregação na metrópole: o Rio de Janeiro em tempo de crise. 2. ed. Rio de Janeiro : Letra Capital, 2015.

LOZANO, Angélica Lozano; GRANADOS, Francisco; GUZMÁN, Alejandro. Impacts of modifications on urban road infrastructure and traffic management: a case study. Procedia Social and Behavioral Sciences. 162, 2014. pp. $368-377$.

MINISTÉRIO DO MEIO AMBIENTE. MMA. Os 25 anos do gerenciamento costeiro no Brasil: Plano Nacional de Gerenciamento Costeiro (PNGC). Brasília: MMA, 2014.

NICOLODI, João Luiz; ZAMBONI, Ademilson Josemar; BARROSO, Gilberto Fonseca. Gestão integrada de bacias hidrográficas e zonas costeiras no Brasil: implicações para a região hidrográfica Amazônica. 2009.

OLIVEIRA, Leise Kellide; CORREIA, Vagner de Assis. Proposta metodológica para avaliação dos benefícios de um centro de distribuição urbano para mitigação dos problemas de logística urbana. J. Transp. Lit. [online]. 2014, vol.8, n.4, pp. 109-145.

PACIONE, Michael. Urban environmental quality and human Wellbeing: a social geographical perspective. Landscape and Urban Planning. 65, 2003. pp. 19-30.

PADILLA, NoeliaAymara. Indicadores ambientales como medidores del impacto de laurbanizaciónenel litoral de Mar delSud, Argentina. Costas. Revista Iberoamericana de Manejo Costero Integrado, v. 2, n. 2, 2013. pp. 88-96.

POLETTE, M.; LINS-DE-BARROS, F.. Os desafios urbanos na zona costeira brasileira frente as mudanças climáticas. Costas 1 (1), 2012. pp.165-180.

SANTA CATARINA. Implantação do Plano Estadual de Gerenciamento Costeiro: plano de gestão regional. 2010.

SANTOS, Marco Pais Neves dos. As novas dinâmicas da sustentabilidade urbana em territórios de pobreza e exclusão social: o caso da Cova da Moura. Revista INVI [online]. 2014, vol.29, n.81, pp. 115-155.ISSN 0718-8358. 
SEN, Amartya Kumar. Desenvolvimento como liberdade. São Paulo: Cia das Letras, 2000.

SILVA, Rafael da. Desenho institucional e promoção da justiça em espaços participativos: implicações em um estudo de caso. Opin. Publica [online]. 2014, vol.20, n.2, pp. 252272.ISSN 1807-0191.

SREEJA, K.G; MADHUSOODHANAN, C.G.; ELDHOT.I..Coastal zones in integrated river basin management in the West Coast of India: Delineation, boundary issues and implications. Ocean \& Coastal Management. 119, 2016. pp. 1-13.

STEIN, Carlos Scartasciniand Ernesto; TOMMASI, Mariano. Political institutions, intertemporal cooperation, and the quality o public policies. Journal of Applied Economics. Vol XVI, No. 1 ,maio, 2013. pp. 1-32.

SVARSTAD, $H$. et. al. Discursive biases of the environmental research framework DPSIR. Land Use Policy, 25(1), 116-125, 2008.

UNITED NATIONS HUMAN SETTLEMENTS PROGRAMME, UN-HABITAT.State of the Word's Cities 2012/2013.New York, 2012.207 p.

WANG, Yafei; et. al. Effect of ecosystem services provided by urban green infrastructure on indoor environment: A literature review. Building and Environment. 77 (2014) 88-100.

WONG, Cecilia. A framework for 'City Prosperity Index': Linking indicators, analysis and policy. Habitat International, 45, 2015. pp 3-9.

ZHANG, Xiaoling, et. al.Sustainable infrastructure projects in balancing urban rural development: towards the goal of efficiency and equity. Journal of Cleaner Production. 107, 2015. pp. $445-454$.

ZHANG, Y., LIU, H.; FATH, B. D. Synergism analysis of an urban metabolic system: Model development and a case study for Beijing, China. Ecological Modelling, 272, 2014. pp. 188197.

\section{NOTAS DE AUTOR}

\section{CONTRIBUIÇÃO DE AUTORIA}

Os papéis descrevem a contribuição específica de cada colaborador para a produção acadêmica inserir os dados dos autores conforme exemplo, excluindo o que não for aplicável.

Nilton Manoel Lacerda Adão - Concepção. Coleta de dados, Análise de dados, Elaboração do manuscrito, revisão e aprovação da versão final do trabalho

Marcus Polette - Concepção e elaboração do manuscrito. Coleta de dados Participação ativa da discussão dos resultados; Revisão e aprovação da versão final do trabalho.

\section{FINANCIAMENTO}

Não se aplica.

\section{CONSENTIMENTO DE USO DE IMAGEM}

Não se aplica.

APROVAÇÃO DE COMITÊ DE ÉTICA EM PESQUISA

Não se aplica. 
LICENÇA DE USO

Este artigo está licenciado sob a Licença Creative Commons CC-BY. Com essa licença você pode compartilhar, adaptar, criar para qualquer fim, desde que atribua a autoria da obra.

HISTÓRICO

Recebido em: 20-02-2016

Aprovado em: 09-10-2019 